4. McCallum M, De Marco A, Lempp FA, Tortorici MA, Pinto D, Walls AC, et al. N-terminal domain antigenic mapping reveals a site of vulnerability for SARS-CoV-2. Cell. 2021;184:23322347.e16. https://doi.org/10.1016/j.cell.2021.03.028

5. Harvey WT, Carabelli AM, Jackson B, Gupta RK, Thomson EC, Harrison EM, et al.; COVID-19 Genomics UK (COG-UK) Consortium. SARS-CoV-2 variants, spike mutations and immune escape. Nat Rev Microbiol. 2021;19:409-24. https:/ / doi.org/10.1038/s41579-021-00573-0

6. Verghese M, Jiang B, Iwai N, Mar M, Sahoo MK, Yamamoto F, et al. A SARS-CoV-2 variant with L452R and E484Q neutralization resistance mutations. J Clin Microbiol. 2021;59:e0074121. https://doi.org/10.1128/JCM.00741-21

7. Edara VV, Pinsky BA, Suthar MS, Lai L, Davis-Gardner ME, Floyd K, et al. Infection and vaccine-induced neutralizingantibody responses to the SARS-CoV-2 B.1.617 variants. N Engl J Med. 2021;NEJMc2107799. https:// doi.org/10.1056/ NEJMc2107799

8. Greaney AJ, Loes AN, Crawford KHD, Starr TN, Malone KD, Chu HY, et al. Comprehensive mapping of mutations in the SARS-CoV-2 receptor-binding domain that affect recognition by polyclonal human plasma antibodies. Cell Host Microbe. 2021;29:463-476.e6. https:// doi.org/10.1016/j.chom.2021.02.003

9. Moir S, Fauci AS. B cells in HIV infection and disease. Nat Rev Immunol. 2009;9:235-45. https://doi.org/10.1038/nri2524

Address for correspondence: Benjamin A. Pinsky, Stanford University School of Medicine, 3375 Hillview Ave, Rm 2913, Palo Alto, CA 94304, USA; email: bpinsky@stanford.edu

\section{SARS-CoV-2 Delta Variant among Asiatic Lions, India}

\author{
Anamika Mishra, Naveen Kumar, \\ Sandeep Bhatia, Ashutosh Aasdev, \\ Sridhar Kanniappan, Abelraj Thaya Sekhar, \\ Aparna Gopinadhan, Ramu Silambarasan, \\ Chirukandoth Sreekumar, Chandan Kumar Dubey, \\ Meghna Tripathi, Ashwin Ashok Raut, \\ Vijendra Pal Singh
}

\footnotetext{
Author affiliations: ICAR-National Institute of High Security Animal Disease, Bhopal, India (A. Mishra, N. Kumar, S. Bhatia, A. Aasdev, C.K. Dubey, M. Tripathi, A.A. Raut, V.P. Singh); Arignar Anna Zoological Park, Chennai, India (S. Kanniappan, A.T. Sekhar, A. Gopinadhan, R. Silambarasan); Madras Veterinary College, Chennai (C. Sreekumar); All India Institute of Medical Sciences, Bhopal (A.A. Raut)
}

DOI: https://doi.org/10.3201/eid2710.211500
In May 2021, severe acute respiratory syndrome coronavirus 2 (SARS-CoV-2) was detected in Asiatic lions in a zoological park in India. Sequence and phylogenetic analyses showed the SARS-CoV-2 strains were the B.1.617.2 (Delta) variant. To reduce transmission of variants of concern, surveillance of SARS-CoV-2 in wild animal populations should be increased.

Cevere acute respiratory syndrome coronavirus 2 $\checkmark$ (SARS-CoV-2), in natural conditions, has shown a broad host susceptibility range (1). Identifying susceptible animal species, reservoirs, and cross-species transmission is a global scientific and public health concern. We found evidence of natural SARS-CoV-2 infection in Asiatic lions (Panthera leo persica) caused by the lineage B.1.617.2 (Delta) variant (World Health Organization nomenclature). We provide coronavirus disease (COVID-19) case information and detailed genomic characterization.

Arignar Anna Zoological Park in Chennai, India, houses 13 Asiatic lions, 9 in a lion safari and 2 each in separate moat enclosures. Beginning May 21, 2021, four of the safari lions started showing signs of loss of appetite, nasal discharge, and occasional coughing. Nasal swab, rectal swab, and fecal samples were collected from 11 lions during May 24-29, 2021, and sent to the Indian Council of Agricultural Research-National Institute of High Security Animal Diseases (Bhopal, India) for molecular investigations (Appendix Table 1, https://wwwnc.cdc.gov/EID/ article/27/10/21-1500-App1.pdf).

We used the VIRALDTECT II Multiplex Real Time PCR Kit for COVID-19 (Genes2Me, https:/ / genes2me. com) to confirm SARS-CoV-2 in 9/11 lions. The other 2 lions were sampled on June 19, 2021, and tested negative for SARS-CoV-2. We also used a World Organisation for Animal Health-recommended reverse transcription PCR (RT-PCR) method to test for canine distemper virus on samples from all 13 lions; all tested negative (2). Two of the infected lions died of COVID-19, one on June 3 and the other on June 16, 2021.

After we confirmed SARS-CoV-2 infection, we performed whole-genome sequencing directly from nasal swab specimens of 4 lions that initially showed symptoms by using the MinION sequencing platform $(\mathrm{Ox}-$ ford Nanopore Technologies, https://nanoporetech. com) (Appendix). We deposited sequences in GenBank (accession nos. MZ363851-4) and GISAID (https:// www.gisaid.org; accession nos. EPI_ISL_2821077-80).

To elucidate the temporal dynamics of SARS-CoV-2 among the lions, we downloaded 310 complete SARSCoV-2 genomes from GISAID (3) that had high coverage and were sequenced from the Tamil Nadu state of 
India, where the zoological park is located, during January 1-June 11, 2021. To generate a set of representative sequences, we used a UCLUST algorithm (4) to select sequences that clustered at the $99.9 \%$ identity threshold. We used MAFFT version 7.475 (5) to align representative SARS-CoV-2 sequences from GISAID with sequences from the lions; then we constructed a phylogenetic tree by using the general time reversible plus gamma model in RAxML version 8.2.12 (6) (Figure).

The amino acid substitutions and deletions in the spike protein of SARS-CoV-2 in lions typically matched with the SARS-CoV-2 Delta variant (Appendix Table 2). We noted amino acid changes in the $\mathrm{N}$ terminal domain (NTD), including T19R, G142D, E156del, F157del, R158G; in the receptor binding motif (RBM), including L452R and T478K; and in D614G of subdomain 2. We also noted a substitution close to S1/ S2 protease cleavage site at P681R and heptad repeat 1 at D950N (Appendix Figures 1, 2). In addition, the lion sequences had the K77T substitution in the NTD, which has been detected in SARS-CoV-2 genomes from 24 countries. In India, frequency of the K77T substitution generally is low $(0.44 \%)$ but occurred in $27.42 \%$ $(65 / 237)$ of sequences in the B.1.167.2 lineage collected in Tamil Nadu state (Appendix Table 2).

The changes in the spike protein, including E156del, F157del, and R158G, of lion sequences were not found in human SARS-CoV-2 sequences from the same geographic area, nor were changes in nonstructural protein 3 (NS3) V88I, possibly because SARS-CoV-2 sequencing is limited in the region. Furthermore, these changes in spike and NS3 were not seen in previously reported lion SARS-CoV-2 sequences, ruling out the possibility that these are host-adapted mutations (7) (Appendix Figure 3). Further investigations could delineate whether changes in the spike protein, namely E156del, F157del, R158G, and K77T, are escape mutants or are associated with increased transmissibility or pathogenicity.

A nucleotide similarity comparison of the 4 lion SARS-CoV-2 sequences against the sequences available in GISAID and phylogenetic analysis revealed that the lion sequences closely matched with a representative human SARS-CoV-2 sequence of B.1.617.2 lineage, GISAID accession no. EPI_ISL_2463770, that comprises 152 viral genome pools collected from the same geographic region during the same month that the lions' samples were collected (Figure; Appendix Figure 4). The park's management strictly adhered to COVID-19 guidelines and did not introduce any new animals to the zoo during India's widespread COVID-19 pandemic. The primary source of SARS-CoV-2 infection in the lions might have been an asymptomatic or paucisymptomatic person. Among the 9 infected lions, 7 were in the lion safari and shared a common habitat, shelter, feeding spaces, and water sources. The other 2 infected lions were on display in separate enclosures that shared a common moat. Because shared habitats offered opportunities for close physical contact, identifying genetically identical SARS-CoV-2 infections in these lions in a short period of time indicates the possibility of lion-to-lion transmission.

In conclusion, evidence of confirmed natural SARS-CoV-2 Delta variant infections in Asiatic lions in India justifies need for increased SARS-CoV-2 surveillance in wild animal species. In addition, strict biosecurity measures should be implemented for wild animals kept in captivity.

This article was preprinted at https:/ / doi.org/10.1101/ 2021.07.02.450663.

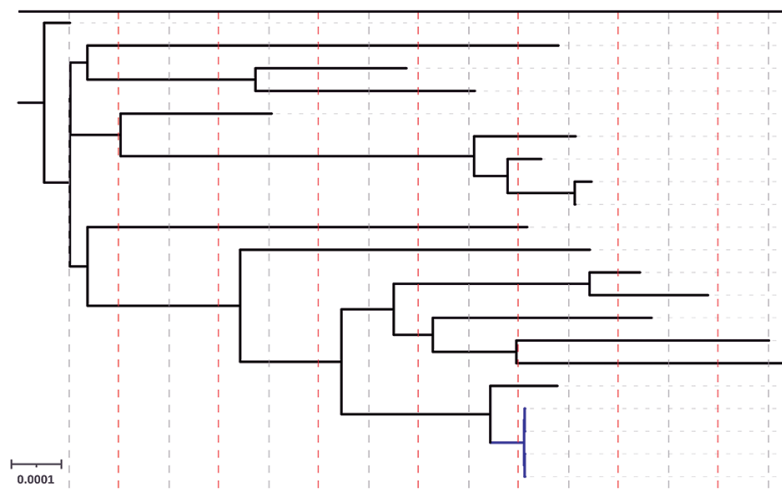

Figure. Complete genome phylogenetic analysis of severe acute respiratory syndrome coronavirus 2 (SARS-CoV-2) detected in Asiatic lions (Panthera leo persica), India (blue text), and representative sequences of different clusters generated at $99.9 \%$ identity threshold from the available SARS-COV-2 sequences from Tamil Nadu, India, in the GISAID. The maximum-likelihood tree was rooted to WuhanHu-1 reference sequence (GISAID accession no. EPI_ISL_402124). GenBank accession numbers are provided for the sequences from this study. Pink numbers in parentheses indicate the number of SARS-CoV-2 genome sequences clustered at $99.9 \%$ identity threshold. Other text colors represent SARS-CoV-2 variants. Scale bar indicates nucleotide substitutions per site. 


\section{Acknowledgments}

We thank Debasis Jana (Arignar Anna Zoological Park, Chennai, India) for providing the clinical information and for sending samples for SARS-CoV-2 molecular investigations for the lions. We also thank V.P. Singh for providing infrastructure facilities to carry out this study, Atul Kumar Pateriya for assistance with quantitative reverse transcription PCR, and the originating and submitting laboratories for sharing SARS-CoV-2 genomic sequence data via GISAID.

This study was funded by the service project of ICARNational Institute of High Security Animal Diseases (ICARNIHSAD), Bhopal, India, and ICAR-National Agricultural Science Fund (grant no. NASF/ABA-8027/2020-21).

\section{About the Author}

Dr. Mishra holds a senior scientist position in the Zoonotic Diseases Group, ICAR-National Institute of High Security Animal Diseases, Bhopal, India. Her primary research interests are the pathogenomics and host-pathogen interactions of high-risk zoonotic viruses.

\section{References}

1. World Organisation for Animal Health. SARS-COV-2 in animals - situation report 1 [cited 2021 Jun 16]. https://www.oie.int/app/uploads/2021/06/sars-cov-2situation-report-1.pdf

2. Frisk AL, König M, Moritz A, Baumgärtner W. Detection of canine distemper virus nucleoprotein RNA by reverse transcription-PCR using serum, whole blood, and cerebrospinal fluid from dogs with distemper. J Clin Microbiol. 1999;37:3634-43. https:/ / doi.org/10.1128/ JCM.37.11.3634-3643.1999

3. Elbe S, Buckland-Merrett G. Data, disease and diplomacy: GISAID's innovative contribution to global health. Glob Chall. 2017;1:33-46. https:/ / doi.org/10.1002/gch2.1018

4. Edgar RC. Search and clustering orders of magnitude faster than BLAST. Bioinformatics. 2010;26:2460-1. https:/ / doi.org/10.1093/bioinformatics/btq461

5. Katoh K, Standley DM. MAFFT multiple sequence alignment software version 7: improvements in performance and usability. Mol Biol Evol. 2013;30:772-80. https:/ / doi.org/ 10.1093/molbev/mst010

6. Stamatakis A. RAxML version 8: a tool for phylogenetic analysis and post-analysis of large phylogenies. Bioinformatics. 2014;30:1312-3. https:/ / doi.org/10.1093/ bioinformatics/btu033

7. McAloose D, Laverack M, Wang L, Killian ML, Caserta LC, Yuan F, et al. From People to Panthera: natural SARS-CoV-2 infection in tigers and lions at the Bronx Zoo. MBio. 2020;11:e02220-20. https://doi.org/10.1128/ mBio.02220-20

Address for correspondence: Anamika Mishra, Pathogenomics Lab, Zoonotic Diseases Group, ICAR-National Institute of High Security Animal Disease, Bhopal 462022, India; email: reach2anamika@yahoo.com

\section{SARS-CoV-2 Variants in Immunocompromised Patient Given Antibody Monotherapy}

\author{
Aurélie Truffot, Julien Andréani, Marion Le Maréchal, \\ Alban Caporossi, Olivier Epaulard, Raphaele Germi, \\ Pascal Poignard, Sylvie Larrat \\ Author affiliation: Centre Hospitalier Universitaire Grenoble Alpes, \\ Grenoble, France
}

\section{DOI: https://doi.org/10.3201/eid2710.211509}

\begin{abstract}
A 72-year-old immunocompromised man infected with severe acute respiratory syndrome coronavirus 2 received bamlanivimab monotherapy. Viral evolution was monitored in nasopharyngeal and blood samples by melting curve analysis of single-nucleotide polymorphisms and whole-genome sequencing. Rapid emergence of spike receptor binding domain mutations was found, associated with a compartmentalization of viral populations.
\end{abstract}

A 72-year-old immunocompromised man in France who had chronic lymphocytic leukemia associated with hypogammaglobinemia for 4 years experienced diarrhea, asthenia, fever, and cough associated with coronavirus disease (COVID-19). Although he had received 1 injection of severe acute respiratory syndrome coronavirus 2 (SARS-CoV-2) mRNA vaccine (BNT162b2; Pfizer/BioNTech, https://www.pfizer.com) 20 days earlier, we confirmed a diagnosis of COVID-19 by using a semiquantitative SARS-CoV-2 reverse transcription PCR (RT-PCR) viral load assay. This assay showed a cycle threshold $\left(C_{t}\right)$ value of 27 for a nasopharyngeal swab specimen. His most recent monoclonal antibody $(\mathrm{mAb})$ chemotherapy treatment (venetoclax and rituximab) had been conducted 17 days earlier. Because of his immunocompromised status, treatment with bamlanivimab (LY-CoV555), a neutralizing IgG1 mAb, was initiated at day 0, 4 days after onset of symptoms (Table). The patient received an infusion of $700 \mathrm{mg}$ in a single dose and was discharged.

Analysis of samples showed a high viral load in a nasopharyngeal swab specimen $\left(C_{t} 20\right)$ and a blood sample $\left(C_{t} 37\right)$ (Table). Three days after the mAb infusion, the patient's symptoms worsened, and he was hospitalized in the Infectious Diseases Department at Grenoble Hospital (Grenoble, France) on day 6. The condition of the patient had deteriorated; he had an additional need for oxygen, which resulted in a convalescent-phase plasma transfusion on day 10 .

After this treatment, the condition of the patient continued to deteriorate, and he was transferred to 